\title{
Influence of publication year, add-on design, and geographical region on the progression of Alzheimer's disease: a modeling analysis of literature aggregate data
}

\section{CURRENT STATUS: UNDER REVIEW}

Alzheimer's Research \& Therapy $\mathbf{B M C}$

ningyuan zhang

Shanghai University of traditional Chinese Medicine

ORCiD: https://orcid.org/0000-0002-9376-2658

Xijun Zheng

Shanghai University of Traditional Chinese Medicine

Hongxia Liu

Shanghai University of Traditional Chinese Medicine

Qingshan Zheng

qingshan.zheng@drugchina.netCorresponding Author

Lujin Li

Shanghai University of Traditional Chinese Medicine

\section{DOI:}

10.21203/rs.3.rs-17692/v1

\section{SUBJECT AREAS}

Cognitive Neuroscience

\section{KEYWORDS}

Alzheimer's disease, disease progression model, model-based meta-analysis, modelling and simulation 
Abstract

Background Our objective was to develop a disease progression model for cognitive decline in Alzheimer's disease $(A D)$ and to determine whether disease progression of $A D$ is related to the year of publication, add-on trial design, and geographical regions.

Methods Placebo-controlled randomized AD clinical trials were systemically searched in public databases. Longitudinal placebo response (mean change from baseline in the cognitive subscale of the Alzheimer's Disease Assessment Scale [ADAS-cog]) and the corresponding demographic information were extracted to establish a disease progression model. Covariate screening and subgroup analyses were performed to identify potential factors affecting the disease progression rate. Results A total of 142 publications (148 trials) were included in this model-based meta-analysis. The typical disease progression rate was 5.82 points per year. The baseline ADAS-cog score was included in the final model using an inverse-U type function. Age was found to be negatively correlated with disease progression rate. After correcting the baseline ADAS-cog score and the age effect, no significant difference in disease progression rate was found between trials published before and after 2008, and between trials using add-on design and those that did not use add-on design. However, a significant difference was found among different trial regions. Trials in East Asian countries showed the slowest decline rate and the largest placebo effect.

Conclusions Our model successfully quantified AD disease progression by integrating baseline ADAScog score and age as important predictors. These factors and geographic location should be considered when optimizing future trial designs and conducting indirect comparisons of clinical outcomes.

\section{Full Text}

Due to technical limitations, full-text HTML conversion of this manuscript could not be completed. However, the manuscript can be downloaded and accessed as a PDF. Figures 


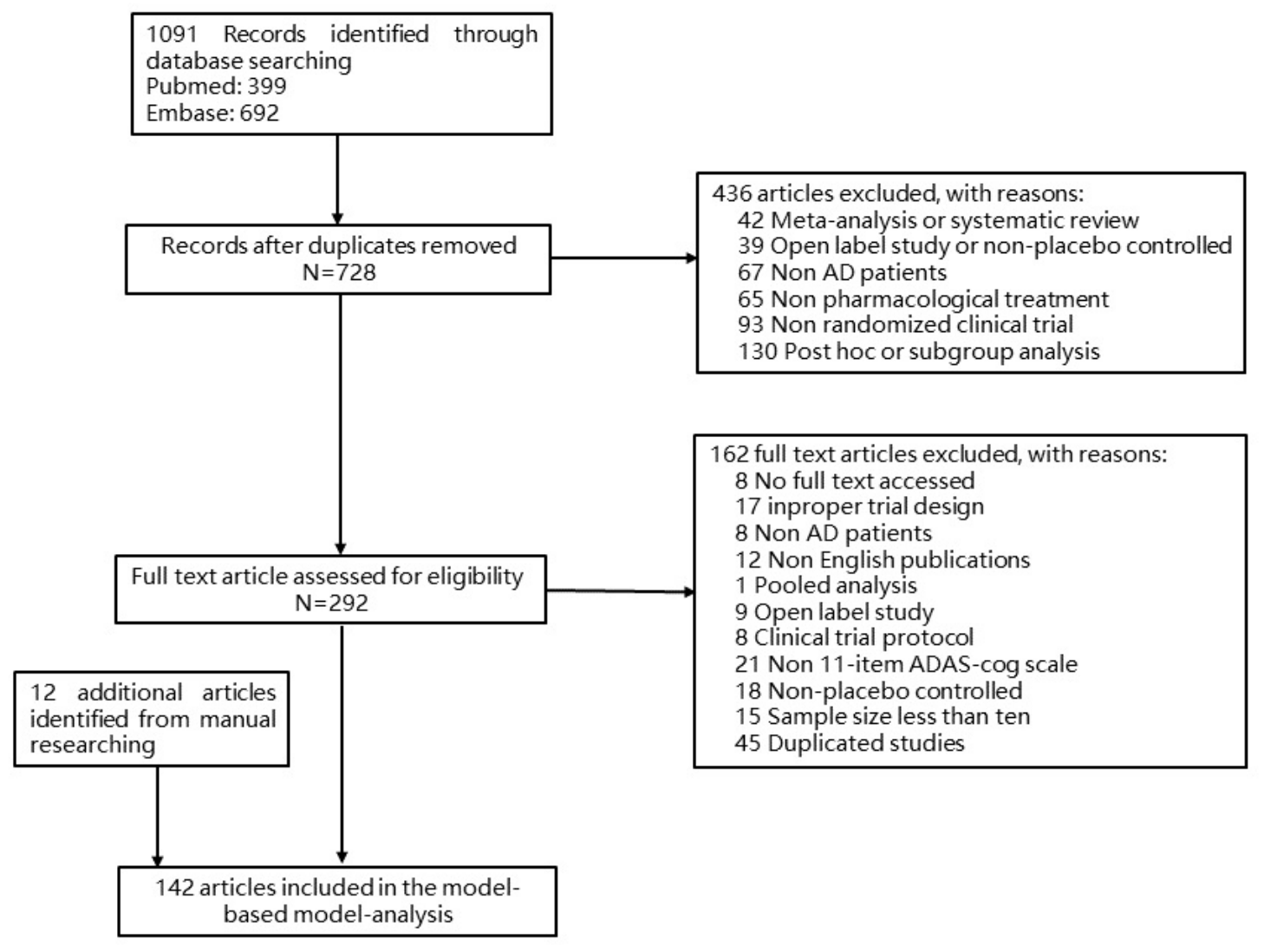

Figure 1

The flow diagram illustrating the inclusion and exclusion of studies into the final analysis. 
(A)

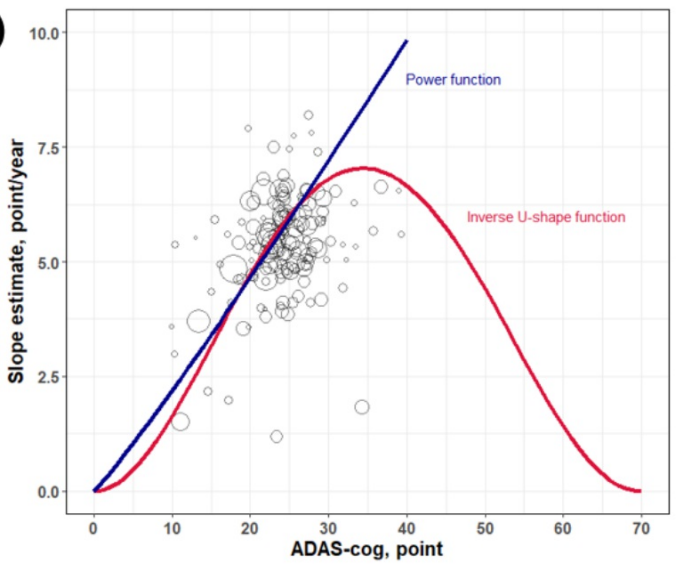

(C)

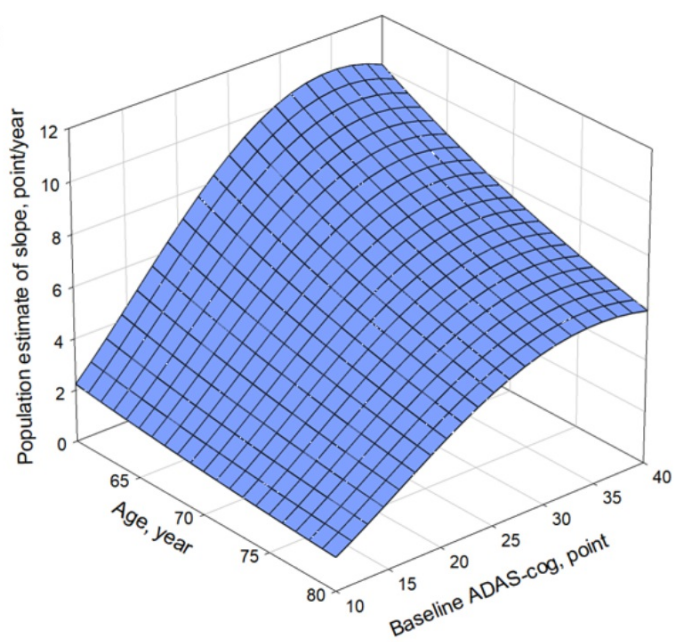

(B)

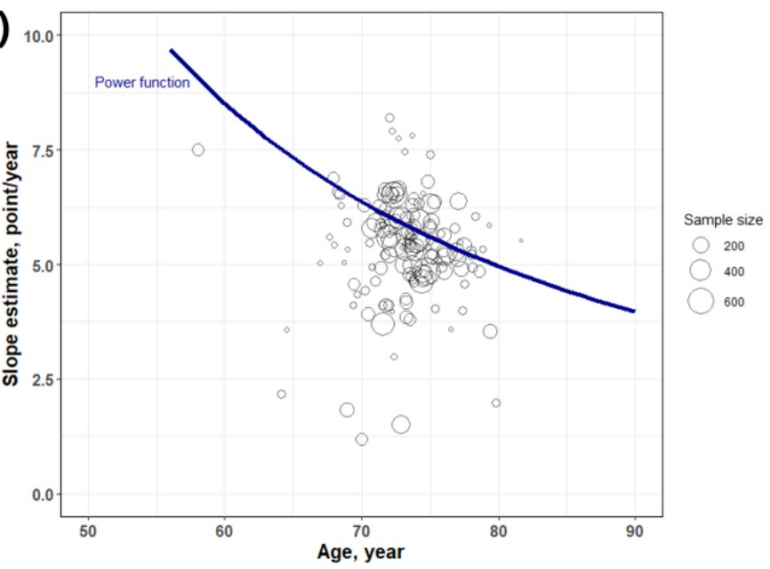

Figure 2

(A) Plot of the individual estimate of disease progression rate $\alpha$ derived from the base model versus the baseline ADAS-cog score. The two parameter-covariate relationships, power and U-type function, were demonstrated by using blue and red lines, respectively. (B) Plot of the individual estimate of disease progression rate $\alpha$ derived from the base model versus the baseline age. The blue fitted line demonstrated the correlation between them. In plot (A) and (B), each black circle stands for one independent study and the circle size is proportional to the corresponding sample size. (C) A surface diagram to describe the relationship among age, ADAS-cog score, and the disease progression rate. Younger patients with higher baseline ADAS-cog score were predicted to manifest faster cognitive decline. 
(A)

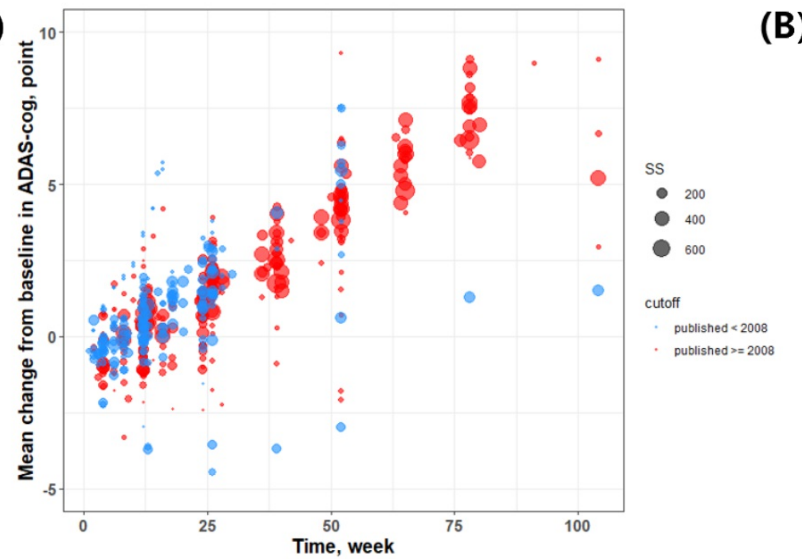

(C)

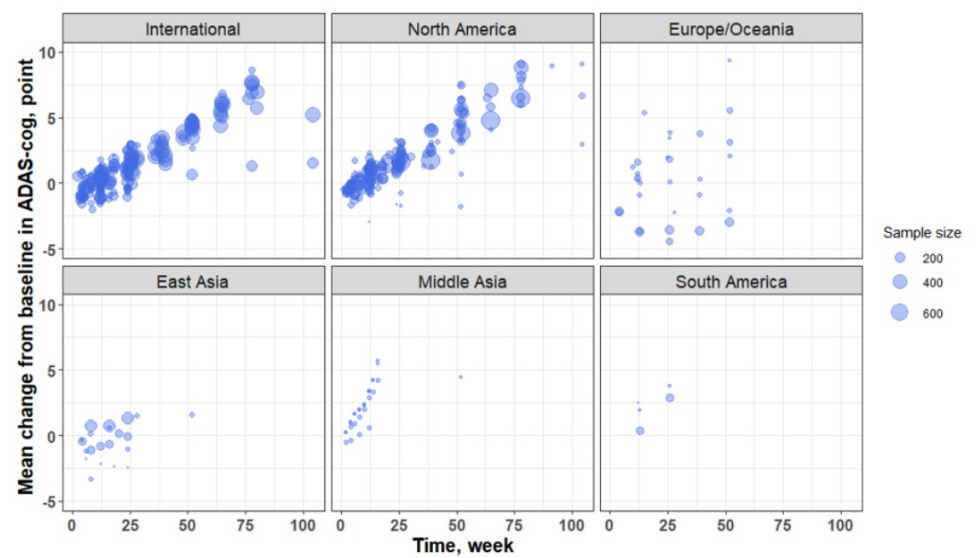

Figure 3

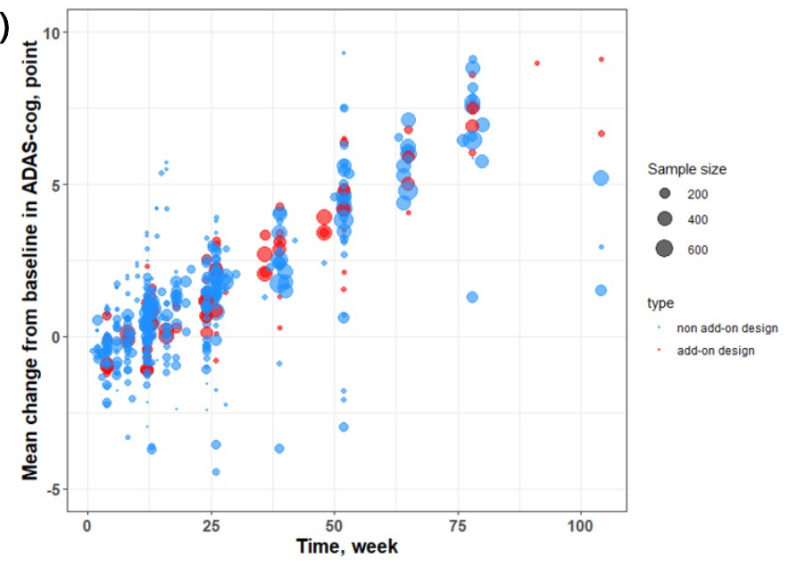


(A)

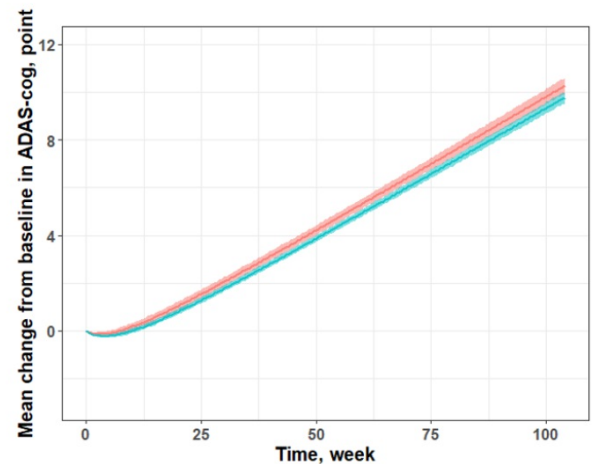

(C)

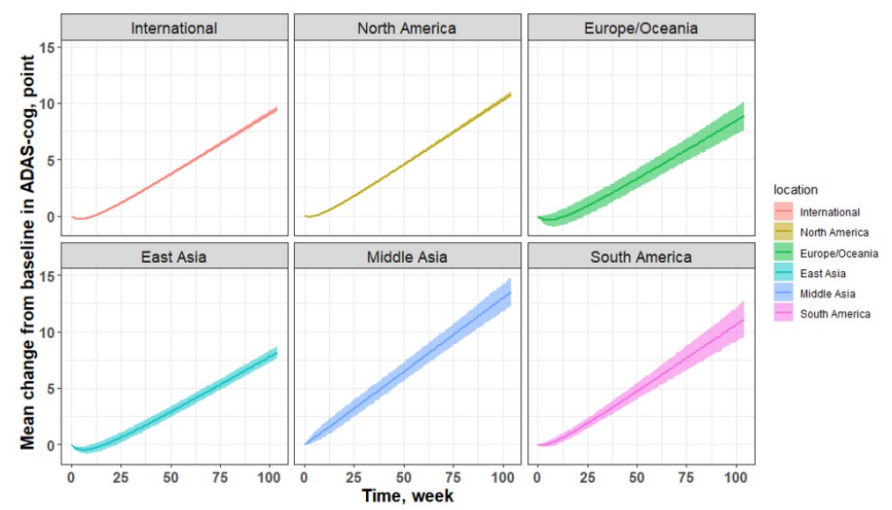

(B)

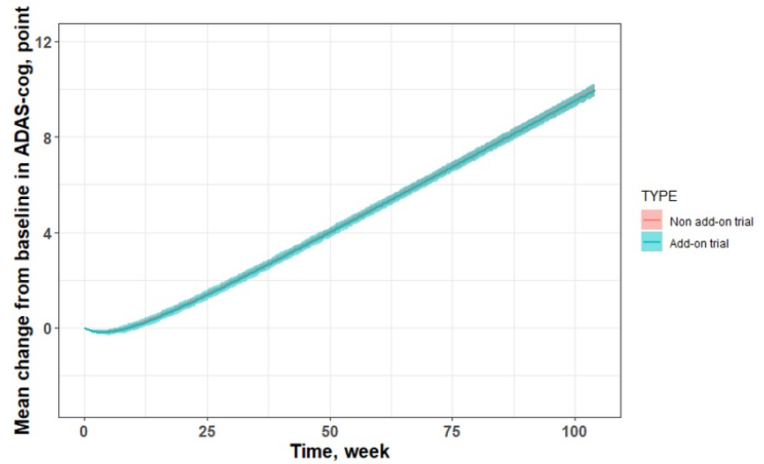

Figure 4

Typical time course of the change of ADAS-cog score from baseline under different stratification scenes: (A) published before 2008 versus after 2008, (B) add-on design versus non add-on design, (C) different geographic locations. The shadows are the model predicted $95 \% \mathrm{Cl}$ of the placebo response. The solid line is the typical value of the model predicted placebo response.

\section{Supplementary Files}

This is a list of supplementary files associated with this preprint. Click to download. additional_file.docx 